\title{
The consequences of a refund in threshold public good games $^{*}$
}

\author{
Edward Cartwright ${ }^{\ddagger} \quad$ Anna Stepanova ${ }^{\S}$
}

5th May 2015

Keywords: Public good, threshold, refund, money back guarantee.

JEL codes: C72, H41, C92

\begin{abstract}
We revisit the question of whether a refund increases efficiency in threshold public good games. New experimental evidence is presented on the effect of endowment size. We demonstrate that a refund increases efficiency if and only if the endowment is small relative to the threshold. We also propose a novel way to analyze the effect of a refund. Specifically, we argue that a refund increases efficiency only if significantly many groups converge towards zero contributions in the absence of a refund.
\end{abstract}

\footnotetext{
${ }^{*}$ We are grateful to Federica Alberti for research assistance and an anonymous referee for comments on an earlier version of the paper. This research was supported by a University of Kent Social Sciences small Faculty Grant 'Threshold public good games and impulse balance theory'.

${ }^{\dagger}$ The final version of this paper appeared in Economics Letters, DOI: 10.1016/j.econlet.2015.05.032, copyright Elsevier.

${ }^{\ddagger}$ School of Economics, University of Kent, UK. Corresponding author, email E.J.Cartwright@kent.ac.uk. ${ }^{\S}$ School of Economics, University of Kent, UK.
} 


\section{Introduction}

Many public goods can be categorized as threshold public goods in the sense that the good is provided if and only if total contributions reach some critical threshold (Taylor and Ward 1982, Hampton 1987). Experimental evidence suggests considerable inefficiency in providing such goods (Croson and Marks 2000). The literature has, therefore, focussed on institutions that can improve efficiency such as voting (Rauchdobler, Sausgruber and Tyran 2010) and a membership fee (Bchir and Willinger 2013).

The institution that has arguably received most attention is that of a refund. Evidence on the effect of refunds is, however, inconclusive. To illustrate, Table 1 (that we shall revisit in the subsequent analysis) details the success rate at providing public goods in all studies that allow direct comparison of treatments with and without a refund. ${ }^{1}$ In many cases the refund makes little difference (Isaac, Schmidtz and Walker 1989, Cadsby and Maynes 1999). Moreover, in those where it does the difference is statistically insignificant (with one exception). ${ }^{2}$ This insignificance cannot be easily dismissed as due to lack of statistical power because the groups most successful at providing the public good are often observed when there is no refund (Coats, Gronberg and Grosskopf 2009).

If a refund only works in certain situations then it is essential to know what these are (Cadsby and Maynes 1999), particularly if refunds would be costly to implement. In this paper we demonstrate that the endowment of players is critical. Combining this insight with those of Isaac et al. (1989) and Cadsby and Maynes (1999) we show that a refund enhances efficiency if and only if endowments are small relative to the threshold. We also propose a general approach to studying the effect of refunds that builds on Isaac et al. (1989). A refund is posited to enhance efficiency only if many groups would converge on zero contributions in the absence of a refund. We feel that these combined insights provide a clear and persuasive story of when a refund makes a difference.

We proceed as follows: In Section 2 we introduce notation, in Section 3 we describe our experimental design, in Section 4 we provide our main results and in Section 5 we conclude. Experiment instructions are provided in an appendix.

\footnotetext{
${ }^{1}$ We restrict attention to simltaneous, continuous choice threshold public good games. (Note that Isaac et al. (1989) have a form of rebate if contributions are above the threshold and Coats et al. (2009) use stranger matching.) For results with sequential choice see Coats et al. (2009). For results with binary choice see Dawes et al. (1986) and Rapoport and Eshed Levy (1989).

${ }^{2}$ Table 1 also details our results, to be discussed below, where we also observe a statistically significant difference.
} 
Table 1: Observed success rates at providing the public good in studies that compare the effect of a refund with no refund. As will be defined in section $2, T$ is threshold, $V$ is the value of the public good, and $E$ is endowment. The number of independent groups or cohorts is given in brackets. We also detail the step return (SR) and endowment multiple $(\mathrm{EM})$, defined in Section 4. * indicates a significant difference at the $10 \%$ level and ${ }^{* *}$ at the $5 \%$ level.

\begin{tabular}{llccccc}
\multicolumn{1}{c}{ Paper } & \multicolumn{1}{c}{ Game } & \multicolumn{2}{c}{ Success rates $(\%)$} & SR & EM \\
& & No refund & Refund & Difference & & \\
\hline Coats et al. & Simultaneous & $26.1(3)$ & $46.7(3)$ & 20.6 & 2.0 & 1.7 \\
\hline Isa09) & & $35.0(6)$ & $41.7(6)$ & 6.67 & 1.2 & 2.3 \\
$(1989)$ & Low $T$ & $26.7(6)$ & $53.3(6)$ & 26.67 & 1.2 & 1.2 \\
& Hedium $T$ & $15.0(6)$ & $56.7(6)$ & $41.67^{*}$ & 1.2 & 1.0 \\
\hline Cadsby & $T=25, V=5$ & $7.7(2)$ & $16.0(1)$ & 8.3 & 2.0 & 4.0 \\
and Maynes & $T=25, V=20$ & $64.1(2)$ & $56.4(2)$ & -7.7 & 8.0 & 4.0 \\
(1999) & $T=50, V=7.5$ & $0.0(2)$ & $28.0(1)$ & 28.0 & 1.5 & 2.0 \\
& $T=50, V=11$ & $12.0(3)$ & $20.0(1)$ & 8.0 & 2.2 & 2.0 \\
& $T=50, V=20$ & $20.3(3)$ & $44.0(1)$ & 23.7 & 4.0 & 2.0 \\
& $T=50, V=30$ & $24.0(2)$ & $60.0(1)$ & 36.0 & 6.0 & 2.0 \\
& $T=75, V=10$ & $8.0(1)$ & $10.0(2)$ & 2.0 & 1.3 & 1.3 \\
& $T=75, V=22.5$ & $0.0(1)$ & $40.0(1)$ & 40.0 & 3.0 & 1.3 \\
& $T=75, V=32.5$ & $16.0(1)$ & $36.0(1)$ & 20.0 & 4.3 & 1.3 \\
\hline Our results & High $E$ & $61.1(9)$ & $64.7(6)$ & -2.4 & 2.0 & 2.8 \\
& Baseline $E$ & $49.5(8)$ & $55.0(8)$ & 5.5 & 2.0 & 2.2 \\
& Low $E$ & $16.4(9)$ & $61.3(6)$ & $44.9 * *$ & 2.0 & 1.2
\end{tabular}

\section{Notation}

A symmetric threshold public good game is characterized by four integers: the number of players $n$, size of endowment $E$, threshold $T<n E$, and value of the public good $V>E$. Each player is endowed with $E$ units of a private good. Simultaneously, players decide how much of their endowment to contribute towards a public good. For each player $i=\overline{1, n}$, let $x_{i} \in\{0,1, \ldots, E\}$ denote the contribution of player $i$. A contribution profile $\left(x_{1}, \ldots, x_{n}\right)$ lists the contribution of each player. Let $Y=\sum_{j=1}^{n} x_{j}$ denote total contributions and let $Y_{-i}=Y-x_{i}$.

In the case of refund the payoff function of player $i=\overline{1, n}$ can be written

$$
\pi_{i}\left(x_{i}, Y_{-i}\right)=\left\{\begin{array}{ll}
E-x_{i}+V & \text { if } x_{i}+Y_{-i} \geq T \\
E & \text { if } x_{i}+Y_{-i}<T
\end{array} .\right.
$$


Thus, if total contributions equal or exceed the threshold $T$ then the public good is provided and each player receives an additional $V$ units of private good. If total contributions are less than the threshold then the public good is not provided and each player receives a refund. ${ }^{3}$ In the case of no refund the payoff function of player $i=\overline{1, n}$ can be written

$$
\pi_{i}\left(x_{i}, Y_{-i}\right)=\left\{\begin{array}{ll}
E-x_{i}+V & \text { if } x_{i}+Y_{-i} \geq T \\
E-x_{i} & \text { if } x_{i}+Y_{-i}<T
\end{array} .\right.
$$

\subsection{Nash Equilibria}

Contribution profile $\left(x_{1}^{*}, \ldots, x_{n}^{*}\right)$ is a strict (pure strategy) Nash equilibrium if and only if $\pi_{i}\left(x_{i}^{*}, Y_{-i}^{*}\right)>\pi_{i}\left(x_{i}, Y_{-i}^{*}\right)$ for all $x_{i} \in\{0,1, \ldots, E\}, x_{i} \neq x_{i}^{*}$ and all $i=\overline{1, n}$. In order to characterize the set of Nash equilibria let $\Lambda$ denote the set of contribution profiles where $\left(x_{1}, \ldots, x_{n}\right) \in \Lambda$ if and only if

$$
\sum_{i \in N} x_{i}=T \text { and } \max _{i \in N}\left\{x_{i}\right\}<V .
$$

In the case of refund the set of strict Nash equilibria is given by $\Lambda$. Thus, any strict Nash equilibrium results in the public good being provided (Bagnoli and Lipman 1989). ${ }^{4}$

In the case of no refund (when $T>\min \{E, V\}$ ) there is an additional strict Nash equilibrium; the absence of a refund means that player $i$ strictly prefers contributing 0 if others contribute 0 . Thus, the set of strict Nash equilibria consists of $\Lambda$ plus the zero vector $(0, \ldots, 0)$. This results in an assurance problem (Isaac et al. 1989); essentially, a player will contribute, in the absence of a refund, only if he expects others to contribute.

\section{Experiment design}

We look at six treatments corresponding to six games, as summarized in Table 2. The Baseline treatment corresponds to a baseline treatment used in the literature (Croson and Marks 2000, Cadsby et al. 2008). The High and Low treatments are motivated and discussed by Alberti and Cartwright (2015). The name High or Low refers to a relatively high or low endowment. To consider the effects of no refund we have three matching NR treatments.

In explaining our experimental design it is important to clarify the differences in our objective and design to that of Alberti and Cartwright (2015). The objective of Alberti and Cartwright (2015) was to study the effect of endowment size in games with a refund. As such, they analyzed the High, Baseline and Low treatments (plus two other treatments not reported here). By contrast, our objective is to analyze the effect of a refund. Hence,

\footnotetext{
${ }^{3}$ We shall assume that if total contributions are above the threshold there is no rebate of the excess contributions. This is standard in the literature, e.g. Cadsby et al. (2008).

${ }^{4}$ If $T>\min \{E, V\}$ there is also a set of non-strict Nash equilibria with no public good provision.
} 
we compare treatments with and without a refund. ${ }^{5}$ This also allows insight on the effect of endowment size in games without a refund.

Table 2: A summary of the experimental treatments. (The three refund treatments are reported by Alberti and Cartwright (2015). The three no refund treatments are not reported elsewhere.)

\begin{tabular}{lllllcc} 
Treatment & $n$ & $E$ & $V$ & $T$ & Refund & No. of groups \\
\hline High NR & 5 & 70 & 50 & 125 & No & 9 \\
High & 5 & 70 & 50 & 125 & Yes & 6 \\
Baseline NR & 5 & 55 & 50 & 125 & No & 8 \\
Baseline & 5 & 55 & 50 & 125 & Yes & 8 \\
Low NR & 5 & 30 & 50 & 125 & No & 9 \\
Low & 5 & 30 & 50 & 125 & Yes & 6
\end{tabular}

The subjects in each experimental session were randomly assigned to a group of 5 , assigned a treatment, and then played the relevant threshold public good game for 25 periods (with a fixed matching protocol). At the end of each period feedback was given on own payoff, total contributions to the public good and whether or not the public good was provided. A total of 230 subjects took part. The experiment was run at the University of Kent (United Kingdom) with subjects recruited from the student population. The experiments were computerized, using z-Tree (Fischbacher 2007). The average payment was $£ 10.25$ and an experimental session lasted about 45 minutes.

\section{Experimental results}

In Figure 1 we plot the average success rate over time for each of the 6 treatments (see also Table 1). Only in the case of a low endowment does the presence of a refund make a significant difference to overall success (Mann-Whitney test of group success over 25 periods, $p=0.02$ for low, $p=0.92$ for baseline, and $p=0.81$ for high) ${ }^{6}$ There is, however, evidence of a dynamic difference in the case of a high endowment. Specifically, the success rate declines over time in the High NR treatment while it increases over time in the High treatment (Mann-Whitney test of change in group success between first 5 and last 5 periods, $p=0.03)$. In the case of a low endowment the success rate increases over time in both treatments but increases significantly more in the Low than Low NR treatment $(p=0.03) .^{7}$

\footnotetext{
${ }^{5}$ To be clear, the data we use for the refund treatments is the same as that reported by Alberti and Cartwright (2015). The data from the matching no refund treatments has not been reported elsewhere (but was collected as part of the same experiment).

${ }^{6}$ Success over periods 1 to 5 gives $p<0.01$ for low, $p=0.45$ for baseline and $p=0.16$ for high. Success over periods 21 to 25 gives $p=0.04$ for low, $p=0.67$ and $p=0.76$ for high.

${ }^{7}$ There is no evidence of any dynamic differences in the baseline treatment $(p=0.48)$.
} 
Figure 1: Average success rate across the 25 periods distinguishing by treatment.
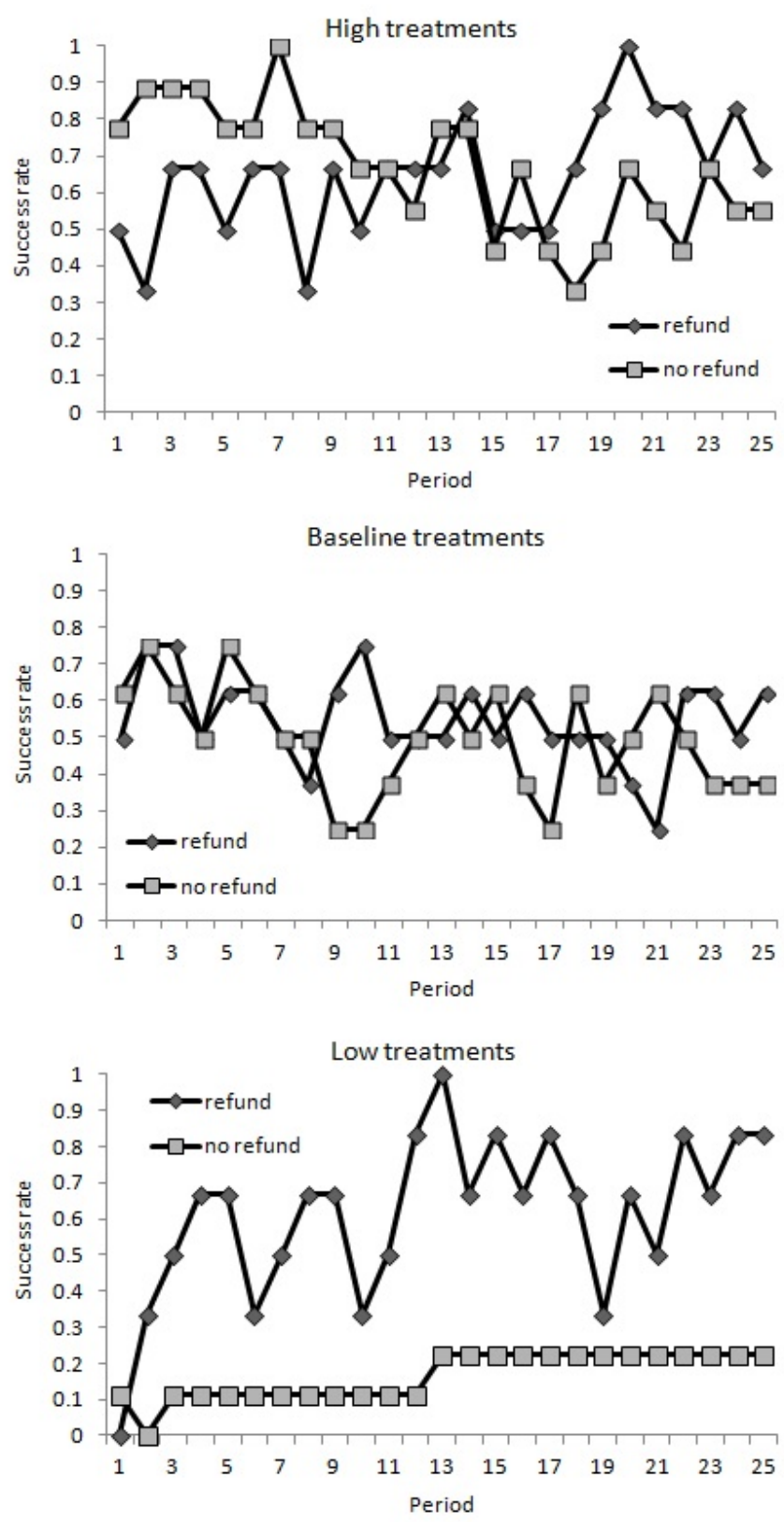
We next look for evidence of convergence towards Nash equilibrium. Specifically, we look at absolute deviation in total contributions from the 'nearest' Nash equilibrium, measured by $\min \{Y,|Y-T|\}$. Figure 2 plots the absolute deviation over time for the 6 treatments. The absolute deviation is significantly declining over time in all treatments except Baseline NR (Mann-Whitney test of group deviation in first 5 periods versus last 5 periods, $p=0.53$ for Baseline NR, $p=0.06$ for High NR, and $p<0.05$ for all other treatments). Only in the case of a low endowment does the presence of a refund make a significant difference ( $p=0.02$ for low, $p=0.10$ for baseline, and $p=0.32$ for high).

\subsection{Endowment multiple}

In summary, the presence of a refund makes a significant difference in the case of a low endowment but no or little difference otherwise. Previous studies (see Table 1) have shown that a refund increases efficiency when there is a high threshold (Isaac et al. 1989, Cadsby and Maynes 1999). But the threshold can be 'high' relative to: (i) the value of the public good, or (ii) endowment. The first possibility is captured by the step return (Croson and Marks 2000)

$$
S R=\frac{V n}{T} .
$$

The second possibility is captured by the endowment multiple (Alberti and Cartwright 2010)

$$
E M=\frac{E n}{T}
$$

Table 1 details the SR and EM for the games considered in the literature. ${ }^{8}$ At first glance EM seems a good indicator of whether a refund will increase efficiency. To formally test this we regressed the difference in success rate between the refund and no refund treatments (weighting according to the number of groups) against SR and EM. The coefficient on SR is insignificant $(2.95, p=0.18)$ but that on EM is highly significant $(-20.1, p<0.001)$. From this we conclude that the presence of a refund increases efficiency if and only if $T$ is high relative to $E$; there is no evidence that $V$ matters. We provide an explanation for this in the next sub-section.

\footnotetext{
${ }^{8}$ The literature has also considered the net reward $V-\frac{T}{n}$ and endowment remainder $E-\frac{T}{n}$ (Cadsby and Maynes 1999, Alberti and Cartwright 2015). We use the step return and endowment multiple here because they are unit free and so make comparison across studies easier.
} 
Figure 2: Absolute deviation from Nash equilibrium across the 25 periods distinguishing by treatment.
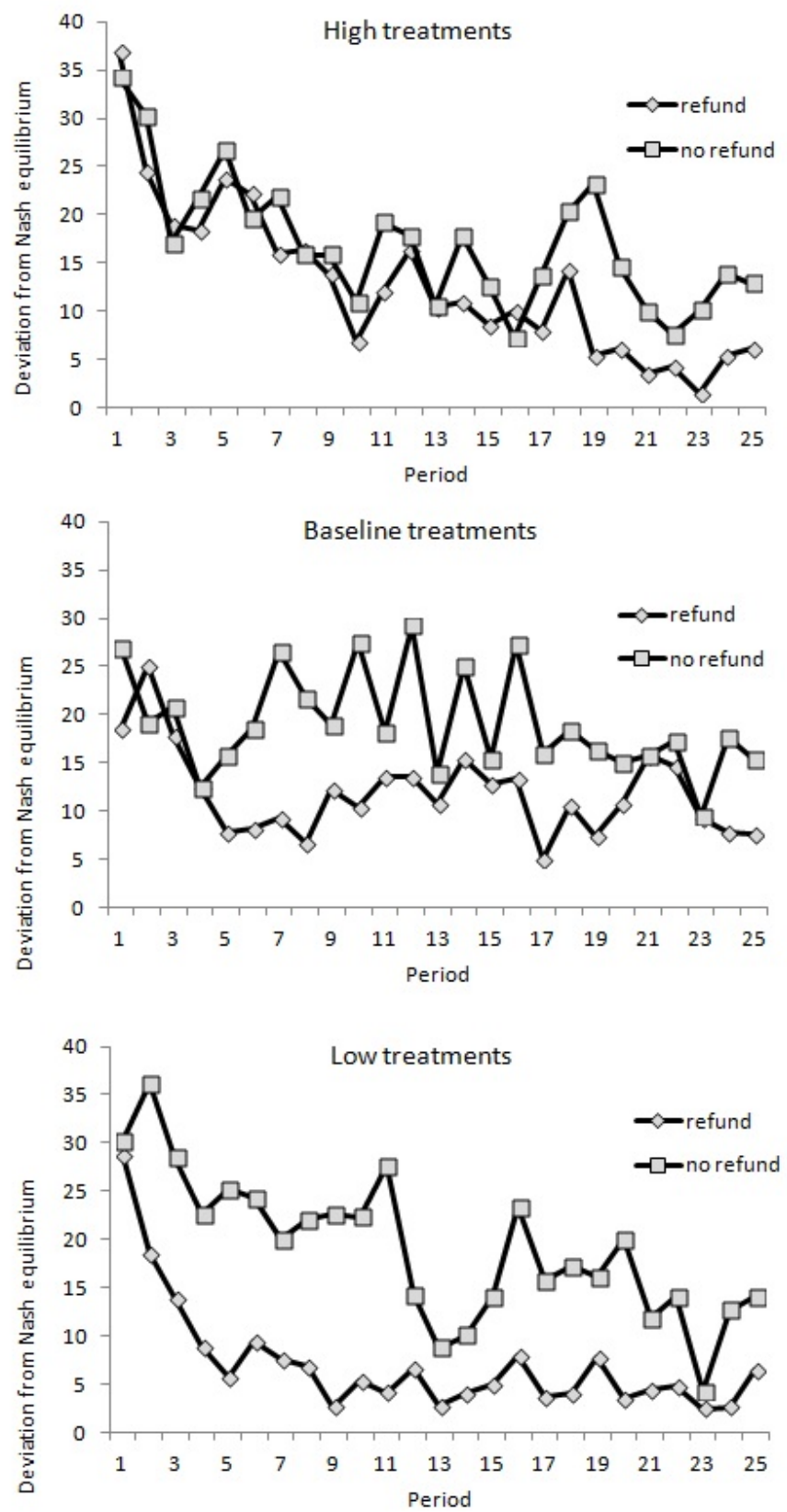


\subsection{Positive and zero groups}

We now propose a general approach that can identify when a refund increases efficiency. Recall that the absence of a refund results in an 'extra' strict Nash equilibrium - the zero vector $(0, \ldots, 0)$. If a group converges on zero contributions then the success rate at providing the public good will clearly be low. This is the essence of the assurance problem (Isaac et al. 1989). In the presence of a refund there is no reason to expect zero contributions. We conjecture, therefore, that any difference in outcome because of a refund is primarily caused by significantly many groups converging on zero contributions in the absence of a refund..$^{9}$

In assessing this conjecture we first remind that the absolute deviation from Nash equilibrium decreased over time suggesting convergence towards Nash equilibrium. Suppose we distinguish between positive and zero groups depending on whether play converges towards set $\Lambda$ or $(0, \ldots 0)$. In practice, we classified a group as positive if $Y>T / 2$ in each of the last five periods and as a zero group if $Y<T / 2$ in each of the last five periods. As summarized in Table 3, this allowed us to classify 43 out of 46 groups.

As expected, zero groups are only observed with no refund. Consistent with our conjecture, the proportion of zero groups in the Low NR treatment is significantly above that in the High NR $(p=0.09$, LR test) and Baseline NR $(p=0.05)$ treatments. Table 4 provides summary statistics on outcomes in positive groups. As conjectured, in positive groups differences in success rate $(p>0.20)$, the dynamics of success $(p>0.11)$, and absolute deviation from Nash equilibrium $(p>0.22)$ are all insignificant (in the high, baseline and low treatments).

Table 3: The number of groups classified as zero or positive groups by treatment.

$\begin{array}{lccc}\text { Treatment } & \text { Zero } & \text { Positive } & \text { Unclassified } \\ \text { High NR } & 2 & 6 & 1 \\ \text { High } & 0 & 6 & 0 \\ \text { Baseline NR } & 1 & 6 & 1 \\ \text { Baseline } & 0 & 8 & 0 \\ \text { Low NR } & 6 & 2 & 1 \\ \text { Low } & 0 & 6 & 0\end{array}$

\footnotetext{
${ }^{9}$ Some differences may still remain. For instance, Alberti, Cartwright and Stepanova (2013) predict a higher success rate when there is no refund in, what we shall shortly call, positive groups (groups that do not converge on $(0, \ldots, 0))$. The differences, however, are predicted to be relatively small.
} 
Table 4: Success rate and absolution deviation from Nash equilibrium in positive groups.

\begin{tabular}{lcccc} 
Treatment & \multicolumn{2}{c}{ Success (\%) } & \multicolumn{2}{c}{ Deviation } \\
& 1 to 25 & 21 to 25 & 1 to 25 & 21 to 25 \\
High NR & 80 & 83 & 19.19 & 12.10 \\
High & 65 & 77 & 12.82 & 4.17 \\
Baseline NR & 66 & 60 & 15.52 & 13.10 \\
Baseline & 55 & 53 & 11.91 & 11.05 \\
Low NR & 70 & 100 & 11.04 & 0.00 \\
Low & 61 & 73 & 7.13 & 4.20
\end{tabular}

All the evidence supports the idea that one can predict whether a refund will make a difference by predicting the likelihood of zero groups. This line of reasoning is useful because, as we discuss shortly, the literature can give insight on the likelihood of zero groups. One thing the literature had neglected is the effect of relative endowment size. Our results provide strong evidence that a relatively small endowment amplifies the assurance problem. An explanation for this is that the smaller is $E$ relatively to $T$ then the 'less affordable' it is for a player to contribute. Put differently, the 'higher is the risk' from contributing. This may decrease willingness to contribute. Moreover, it may decrease expectations that others will contribute.

\section{Conclusion}

We have provided new experimental evidence on whether the presence of a refund increases efficiency in threshold public good games. We demonstrate that a refund increases efficiency only if endowments are low relative to the threshold. A refund, particularly if costly to implement, may, therefore, be inappropriate. This is where our proposed distinction between zero and positive groups proves useful. Wherever convergence on zero contributions is likely to prevail we argue that a refund will make a difference. In this paper we have demonstrated that zero contributions are likely to prevail if the endowment is low relative to the threshold. Other factors may also prove important. For example, Cadsby and Maynes (1998) find that economics and business students are more likely to converge on zero contributions than nurses. Similarly, inequality in endowments has been shown to exasperate the assurance problem (e.g. Tavoni et al. 2011).

\section{References}

[1] Alberti, F. \& E. Cartwright (2015). Does the Endowment of Contributors Make a Difference in Threshold Public Good Games? FinanzArchiv, forthcoming. 
[2] Alberti, F. \& E. Cartwright (2010). Does the Endowment of Contributors Make a Difference in Threshold Public Good Games? University of Kent, School of Economics, Discussion Paper 10/09.

[3] Alberti, F., Cartwright, E., \& Stepanova, A. (2013). Explaining Success Rates at Providing Threshold Public Goods: An Approach Based on Impulse Balance Theory. SSRN working paper number 2309361.

[4] Bagnoli, M., \& Lipman, B. L. (1989). Provision of public goods: Fully implementing the core through private contributions. Review of Economic Studies, 56(4), 583-601.

[5] Bchir, M. A. and M. Willinger (2013). Does a membership fee foster successful public good provision? An experimental investigation of the provision of a step-level collective good. Public Choice 157: 25-39.

[6] Cadsby, C. B., Croson, R., Marks, M., \& Maynes, E. (2008). Step return versus net reward in the voluntary provision of a threshold public good: An adversarial collaboration. Public Choice, 135(3-4), 277-289.

[7] Cadsby, C. B., \& Maynes, E. (1998). Choosing between a socially efficient and freeriding equilibrium: Nurses versus economics and business students. Journal of Economic Behavior $\&$ Organization, 37(2), 183-192.

[8] Cadsby, C. B., \& Maynes, E. (1999). Voluntary provision of threshold public goods with continuous contributions: experimental evidence. Journal of Public Economics, 71(1), 53-73.

[9] Coats, J. C., Gronberg, T. J., \& Grosskopf, B. (2009). Simultaneous versus sequential public good provision and the role of refunds - an experimental study. Journal of Public Economics, 93(1), 326-335.

[10] Croson, R. T., \& Marks, M. B. (2000). Step returns in threshold public goods: A meta-and experimental analysis. Experimental Economics, 2(3), 239-259.

[11] Dawes, R. M., Orbell, J. M., Simmons, R. T., \& Van De Kragt, A. J. (1986). Organizing groups for collective action. American Political Science Review, 80(04), 1171-1185.

[12] Fischbacher, U. (2007). z-Tree: Zurich toolbox for ready-made economic experiments. Experimental economics, 10(2), 171-178.

[13] Hampton, J. (1987). Free-rider problems in the production of collective goods. Economics and Philosophy, 3: 245-273.

[14] Isaac, R. M., Schmidtz, D., \& Walker, J. M. (1989). The assurance problem in a laboratory market. Public choice, 62(3), 217-236. 
[15] Rapoport, A., \& Eshed-Levy, D. (1989). Provision of step-level public goods: Effects of greed and fear of being gypped. Organizational Behavior and Human Decision Processes, 44(3), 325-344.

[16] Rauchdobler, J., Sausgruber, R., and J. R. Tyran (2010). Voting on thresholds for public goods: experimental evidence. FinanzArchiv: Public Finance Analysis, 66(1), 34-64.

[17] Tavoni, A., Dannenberg, A., Kallis, G., \& Löschel, A. (2011). Inequality, communication, and the avoidance of disastrous climate change in a public goods game. Proceedings of the National Academy of Sciences, 108(29), 11825-11829.

[18] Taylor, M., \& Ward, H. (1982). Chickens, whales, and lumpy goods: Alternative models of public-goods provision. Political Studies, 30(3), 350-370. 